\title{
Physical constraints on interacting dark energy models
}

\author{
J. E. Gonzalez ${ }^{1,2, a}$, H. H. B. Silva ${ }^{3, b}$, R. Silva ${ }^{4, c}$, J. S. Alcaniz ${ }^{2,4, d}$ \\ ${ }^{1}$ Departamento de Física, Universidade Federal de Sergipe, Aracaju, SE 49100-000, Brasil \\ 2 Observatório Nacional, Rio de Janeiro, RJ 20921-400, Brasil \\ ${ }^{3}$ Universidade Federal de Campina Grande, Cajazeiras, PB 58900-000, Brasil \\ ${ }^{4}$ Departamento de Física, Universidade Federal do Rio Grande do Norte, Natal, RN 59072-970, Brasil
}

Received: 20 May 2018 / Accepted: 1 September 2018 / Published online: 11 September 2018

(C) The Author(s) 2018

\begin{abstract}
Physical limits on the equation-of-state (EoS) parameter of a dark energy component non-minimally coupled with the dark matter field are examined in light of the second law of thermodynamics and the positiveness of entropy. Such constraints are combined with observational data sets of type Ia supernovae, baryon acoustic oscillations and the angular acoustic scale of the cosmic microwave background to impose restrictions on the behaviour of the dark matter/dark energy interaction. Considering two EoS parameterisations of the type $w=w_{0}+w_{a} \zeta(z)$, we derive a general expression for the evolution of the dark energy density and show that the combination of thermodynamic limits and observational data provide tight bounds on the $w_{0}-w_{a}$ parameter space.
\end{abstract}

\section{Introduction}

The physical mechanism behind the late-time cosmic acceleration is currently one of the major open problems in the field of cosmology. This phenomenon has been evidenced from analysis and interpretation of different observational data sets [1-9] and, in the context of the general relativity theory, can be explained either if one admits the existence of an exotic field, the so-called dark energy, or if the matter content of the universe is subject to dissipative processes $[10,11]$ (see [12-14] for a review).

The lack of knowledge on the nature of the dark sector has motivated several approaches to unveil the physical properties of both dark matter and dark energy. In principle, a thermodynamic analysis should be relevant to constrain the behavior of these dark components or even to restrict

\footnotetext{
a e-mail: javierernesto@on.br

be-mail: heydson.brito@ufcg.edu.br

c e-mail: raimundosilva@ fisica.ufrn.br

de-mail: alcaniz@on.br
}

the range of acceptable values of their parameters. Many approaches of this kind have been formulated in the literature (see, e.g., [15-22] and references therein). For instance, the thermodynamics of a dark energy component described by a varying equation-of-state parameter $(\mathrm{EoS}) \omega=\omega(a)$ with null chemical potential $(\mu=0)$ was discussed in [23] whereas a general treatment for dark energy thermodynamics considering a non-zero chemical potential $(\mu \neq 0)$ was presented in [24], generalising the results of Refs. [15-17,23]. On the other hand, motivated by a possible solution of the so-called coincidence problem $[25,26]$, interacting models of dark matter and dark energy constitute an alternative description of the dark sector which have been largely investigated (see, e.g., [26-35] and references therein). This class of models are based on the premise that there is currently no known symmetry in Nature preventing a non-minimal coupling in the dark sector and, therefore, such possibility as well as its cosmological consequences must be explored. In models of this kind the non-gravitational interactions between the fluids also contribute to their density evolution, thereby violating the usual assumption of adiabaticity (for a recent observational analysis of a large class of interacting models, see [37]).

In this paper, we extend the thermodynamic analyses of $[23,24]$ to a more general framework which assumes a phenomenological energy exchange between the dark energy and the cold dark matter components. Using the approach of $[38,39]$ to obtain the interaction term, we derive the evolution of dark energy density for two equation-of-state (EoS) parameterisations of the type $w=w_{0}+w_{a} \zeta(z)$ [40-42] and impose physical constraints on its parameters from both the second law of thermodynamics and the positiveness of entropy. We also perform a joint statistical analysis using current observational data from distance measurements to type Ia supernovae ( $\mathrm{SNe}$ Ia) from the JLA compilation [43], measurements of $\theta(z)$ obtained from the baryon acoustic oscillations (BAO) signal using the angular two-point correlation 
function (2PACF) [44-47] and the angular acoustic scale of the cosmic microwave background (CMB) provided by the Planck Collaboration 2015 [48]. In our analysis, we also use the latest measurement of the local expansion rate $H_{0}$, as reported in [49]. We show that the usual constraints on the $w_{0}-w_{a}$ parametric space are significantly enhanced when the thermodynamic bounds are incorporated in the observational analysis. Throughout this paper a subscript 0 stands for present-day quantities and a dot denotes time derivative. We assume a flat background and work with units where the speed of light $c=1$.

\section{Interacting models}

First let us consider that the energy-momentum tensor of the cosmic fluid $T^{\mu \nu}$ consists of two perfect fluid parts, i.e.,

$T^{\mu \nu}=T_{1}^{\mu \nu}+T_{2}^{\mu \nu}$,

with $T_{i}^{\mu \nu}=\left(p_{i}+\rho_{i}\right) u^{\mu} u^{\nu}+p_{i} g^{\mu \nu}$, where $\rho_{i}$ is the energy density and $p_{i}$ is the equilibrium pressure of the species $i=1,2$. By considering the Friedmann-LemaitreRobertson-Walker space-time and a coupling between these components, the condition $\nabla_{\nu} T^{\mu \nu}=0$ leads to

$\dot{\rho}_{d m}+3 \frac{\dot{a}}{a} \rho_{d m}=-\dot{\rho}_{x}-3 \frac{\dot{a}}{a}(1+\omega) \rho_{x}=Q$,

where $\rho_{d m}$ and $\rho_{x}$ are the energy densities of cold dark matter (DM) and dark energy (DE), respectively, while $Q$ is the coupling function. For $Q>0$ we have the DE decaying into DM whereas for $Q<0$ the DM component decays into DE.

In the standard context the dark matter density evolves as $\rho_{d m} \propto a^{-3}$. However, if this component interacts with dark energy, such interaction necessarily causes a deviation from standard evolution, which may be characterised by the $\epsilon$ parameter, i.e. $[38,39]$

$\rho_{d m}=\rho_{d m, 0} a^{-3+\epsilon}$,

which is equivalent to a coupling term of the type

$Q=\epsilon H \rho_{d m}$.

where $H=\dot{a} / a$ is the Hubble parameter. In [39], it was shown that the $\epsilon$ parameter must be positive, which means from Eq. (4) that $Q>0$ and, consequently, that the DE decays into DM.

For generality, we consider that the EoS of dark energy is a function of the scale factor, $w(a)$. Replacing this into Eq. (2) one finds $\rho_{x}=\frac{\tilde{\rho}_{x, 0}-\epsilon \rho_{d m, 0} \int \exp \left[3 \int \frac{1+\omega(a)}{a} d a\right] a^{-4+\epsilon} d a}{\exp \left[3 \int \frac{1+\omega(a)}{a} d a\right]}$,

where $\tilde{\rho}_{x, 0}$ is an integration constant and $\omega=\omega(a) \equiv p_{x} / \rho_{x}$ is the time-dependent EoS parameter of dark energy fluid. In order to proceed further, we will assume the following form for the EoS parameter: $\omega(a)=\omega_{0}+\omega_{a} \zeta(a)$, with $\zeta(a)$ obeying two functional forms that has been widely discussed in the literature $[40-42]^{1}$ :

$\zeta(a)=\left\{\begin{array}{l}(1-a) \\ \frac{1-a}{2 a^{2}-2 a+1}\end{array}\right.$

Substituting the above parameterisations into Eq. (5) we find, respectively,

$\frac{\rho_{x}^{(1)}}{\tilde{\rho}_{x, 0}}=\frac{1-A \epsilon \int a^{3\left(\omega_{0}+\omega_{a}\right)+\epsilon-1} \exp \left[3 \omega_{a}(1-a)\right] d a}{a^{3\left(1+\omega_{0}+\omega_{a}\right)} \exp \left[3 \omega_{a}(1-a)\right]}$,

$\frac{\rho_{x}^{(2)}}{\tilde{\rho}_{x, 0}}=\frac{1-A \epsilon \int a^{3 \omega_{0}+\epsilon-1}\left[\frac{a^{2}}{\left(2 a^{2}-2 a+1\right)}\right]^{3 \omega_{a} / 2} d a}{a^{3\left(1+\omega_{0}\right)}\left[\frac{a^{2}}{\left(2 a^{2}-2 a+1\right)}\right]^{3 \omega_{a} / 2}}$,

where $A \equiv \rho_{d m, 0} / \tilde{\rho}_{x, 0}$ is a constant.

Now, considering that the baryonic and radiation components are separately conserved, the Friedmann equation can be written as

$E^{j}(z)=\left[\frac{\Omega_{r}}{a^{4}}+\frac{\Omega_{b}}{a^{3}}+\frac{\Omega_{d m}}{a^{3-\epsilon}}+\tilde{\Omega}_{x} f^{(j)}(a)\right]^{1 / 2}$,

where $E^{j}=H^{j} / H_{0}$, the density parameters follow the usual definition, and $f^{(j)}$ stands for the $\rho_{x}^{(f)} / \tilde{\rho}_{x, 0}$ ratio given by Eqs. (6a) and (6b). Note that the so-called dynamical $\Lambda$ models (see, e.g., [51]) are fully recovered for values of $w_{0}=-1$ and $w_{a}=0$.

\section{Thermodynamic analysis}

In general, the thermodynamic description of the interaction between two perfect fluids requires the knowledge of three quantities: the energy-momentum tensor $T_{i}^{\mu \nu}$, given by Eq. (1), and the particle flow vector $N_{i}^{\mu}$ and the entropy flux $S_{i}^{\mu}$ defined, respectively, as

$N_{i}^{\mu}=n_{i} u^{\mu}$,

$S_{i}^{\mu}=n_{i} \sigma_{i} u^{\mu}$,

where $n_{i} \equiv N_{i} / a^{3}$ is the particle number density and $\sigma_{i} \equiv$ $S_{i} / N_{i}$ the specific entropy (per particle) for each species [52,

\footnotetext{
1 For a recent comparative study between these $w(a)$ parameterisations, we refer the reader to [50].
} 
53]. By considering that the decay into DM or DE affects only the particle mass (the particle number is unaltered), the fluids are composed by variable-mass particles [54]. Therefore, the particle flow vector is conserved as follows

$\nabla_{\mu} N_{i}^{\mu}=\dot{n}_{i}+\Theta n_{i}=0$,

where $\Theta \equiv \nabla_{\mu} u_{i}^{\mu}=3 \dot{a} / a$ is the fluid expansion rate. The specific entropy obeys the Gibbs equation, i.e.,

$n_{i} T_{i} d \sigma_{i}=d \rho_{i}-\frac{\rho_{i}+p_{i}}{n_{i}} d n_{i}$,

Now, assuming that $\rho_{i}=\rho_{i}\left(n_{i}, T_{i}\right)$ and $p_{i}=p_{i}\left(n_{i}, T_{i}\right)$, it can be shown that the temperature evolution law is given by $[23,52,53]$

$\frac{\dot{T}_{i}}{T_{i}}=\left(\frac{\partial p_{0, i}}{\partial \rho_{i}}\right)_{n_{i}} \frac{\dot{n}_{i}}{n_{i}}+\left(\frac{\partial \Pi_{i}}{\partial \rho_{i}}\right)_{n_{i}} \frac{\dot{n}_{i}}{n_{i}}$.

The fact that DM is pressureless means that there is no temperature evolution law for this component. Therefore, only the DE temperature evolution law is relevant for the thermodynamic analysis that follows. The middle and righthand sides of Eq. (2), on the other hand, can be rewritten as $\dot{\rho}_{x}+3\left(\rho_{x}+p_{0}\right) \frac{\dot{a}}{a}=-3 \Pi \frac{\dot{a}}{a}$, where we have split the dark energy pressure into two components: $p_{0}=\omega_{0} \rho_{x}$ and $\Pi$ given by

$\Pi \equiv w_{a} \zeta(a) \rho_{x}+\frac{\epsilon}{3} \rho_{d m}$,

which mimics a fluid with bulk viscosity (see Refs. [23,24] for a discussion). Therefore, the entropy source of the DE fluid is [53]

$\nabla_{\mu} S_{x}^{\mu}=-\frac{\Pi \Theta}{T_{x}}$.

Considering that the DE temperature is always positive and growing in the course of the universe expansion (see, e.g., $[15,23,24])$, the second law of thermodynamics implies that

$\frac{3 \omega_{a} \zeta(a) \rho_{x}}{\epsilon \rho_{d m}} \leq-1$

Along with Eqs. (3), (6a) and (6b), the above inequality provides our first thermodynamic constraint on the DE quantities. For parameterisations (P1) and (P2), they are written, respectively, as

$$
\begin{aligned}
& \omega_{a} \leq-A \frac{\epsilon}{3} \frac{a^{3\left(\omega_{0}+\omega_{a}\right)+\epsilon} \exp \left[3 \omega_{a}(1-a)\right](1-a)^{-1}}{\left\{1-A \epsilon \int a^{3\left(\omega_{0}+\omega_{a}\right)+\epsilon-1} \exp \left[3 \omega_{a}(1-a)\right] d a\right\}}, \\
& \omega_{a} \leq-A \frac{\epsilon}{3} \frac{a^{3 \omega_{0}+\epsilon}\left[\frac{a^{2}}{2 a^{2}-2 a+1}\right]^{3 \omega_{a} / 2}\left(\frac{1-a}{2 a^{2}-2 a+1}\right)^{-1}}{\left\{1-A \epsilon \int a^{3 \omega_{0}+\epsilon-1}\left[\frac{a^{2}}{2 a^{2}-2 a+1}\right]^{3 \omega_{a} / 2} d a\right\}},
\end{aligned}
$$

which clearly are not defined at $a=1$, where $\omega=\omega_{0}$. On the other hand, using the well- known Euler relation with null chemical potential: $T_{x} S_{x}=\left(\rho_{x}+p_{x}\right) V_{x}$ (where $V_{x} \propto a^{3}$ is the comoving volume) the positiveness of entropy ${ }^{2}$ requires that

$[1+\omega(a)] \rho_{x} \geq 0$

which provides our second set of thermodynamic constraints. For parameterisations (P1) and (P2), it is written as

$$
\begin{aligned}
& {\left[1+\omega_{0}+\omega_{a}(1-a)\right] \rho_{x}^{(1)} \geq 0 .} \\
& {\left[1+\omega_{0}+\omega_{a} \frac{1-a}{2 a^{2}-2 a+1}\right] \rho_{x}^{(2)} \geq 0,}
\end{aligned}
$$

respectively. When the dark energy density satisfies the weak energy condition, i.e., $\rho_{x} \geq 0$, for all values of the scale factor $a$ in the interval of study, the second set of thermodynamic constraints is exactly equal to the one obtained for non-interacting models [23,24]:

$[1+\omega(a)] \geq 0$.

For the case in which the dark matter and dark energy components are not coupled $(\epsilon \rightarrow 0)$, one also fully recovers the results of [23] for the both sets of thermodynamic constraints above.

\section{Observational data}

In order to test the class of models discussed in the previous section, we perform a Bayesian statistical ana- lysis using different cosmological observables taking into account the above sets of thermodynamic constraints.

The primary data set used in this analysis is the type Ia supernovae ( $\mathrm{SNe}$ Ia) compilation named Joint Light-curve Analysis (JLA), which comprises 740 observational data obtained by SDSS-II and SNLS collaborations [43]. The distance modulus is standardised using the model

$\hat{\mu}=m_{B}^{*}-\left(M_{B}-\alpha \times X_{1}+\beta \times C\right)$

where $m_{B}^{*}$ is the observed peak magnitude in the rest frame B band, $C$ is the color at the maximum brightness, $X_{1}$ is the

$\overline{2 \text { As stated by }}$ the statistical microscopic concept of entropy: $S=$ $k_{B} \ln W>0$. 
Table 1 Constraints on the cosmological parameters for P1 and P2 considering the Gaussian prior $H_{0}=73.52 \pm 1.62 \mathrm{~km} \mathrm{~s}^{-1} \mathrm{Mpc}^{-1}$

\begin{tabular}{llllll}
\hline & $\epsilon$ & $w_{o}$ & $w_{a}$ & $\Omega_{d m}$ & $H_{0}$ \\
\hline (P1) & $0.010 \pm 0.008$ & $-0.78 \pm 0.14$ & $-1.44 \pm 0.85$ & $0.223 \pm 0.012$ & $71.23 \pm 1.32$ \\
(P2) & $0.011 \pm 0.009$ & $-0.78 \pm 0.12$ & $-1.04 \pm 0.53$ & $0.228 \pm 0.012$ & $71.26 \pm 1.36$ \\
\hline
\end{tabular}

time stretching of the light-curve and $\alpha$ and $\beta$ are nuisance parameters. The absolute magnitude $M_{B}$ is dependent on the host galaxy properties and the effects of this dependence are corrected by the step function:

$M_{B}= \begin{cases}M_{B}^{1} & \text { if } M_{\text {stellar }}<10^{10} M_{\odot}, \\ M_{B}^{1}+\Delta_{M} & \text { if } M_{\text {stellar }} \geq 10^{10} M_{\odot}\end{cases}$

being $M_{\text {stellar }}$ the stellar mass of the SN host galaxy and $\Delta_{M}$ another nuisance calibration parameter [43]. The distance modulus is related to the cosmological model via the luminous distance by

$\mu_{\text {model }}(z)=5 \log \left(\frac{d_{L}(z)}{1 \mathrm{Mpc}}\right)+25$,

where $d_{L}(z)$ is the luminosity distance. Note that both the cosmological and SNe calibration parameters are fitted simultaneously.

In our analysis we also use recent BAO data obtained from a 2-point angular correlation function analysis of the SDSS luminous red galaxies and quasars (hereafter $\theta_{\mathrm{BAO}}$ ) [44-47]. The $\theta_{\mathrm{BAO}}$ data are obtained by measuring the angular separation between pairs for a defined comoving acoustic scale, considering thin redshift shells of order $\delta_{z}=0.01-0.02$. Differently from the usual measurements of the BAO signal obtained from the 2-point correlation function (which assume a fiducial cosmology in order to transform the measured angular positions and redshifts into comoving distances), the 2PACF measurements of $\theta_{\mathrm{BAO}}$ are almost modelindependent, which makes them a robust quantity to test cosmological models. The theoretical value of $\theta_{\mathrm{BAO}}$ for a given cosmology is given by

$\theta_{\mathrm{BAO}}(z)=\frac{r_{s}}{(1+z) d_{A}(z)}$

where $d_{A}=d_{L} /(1+z)^{2}$ and the sound horizon scale is obtained from the expression:

$r_{s}(z)=\frac{1}{\sqrt{3}} \int_{z_{\text {drag }}}^{\infty}\left(1+\frac{3 \Omega_{b}}{4 \Omega_{\gamma}\left(1+z^{\prime}\right)}\right)^{-1 / 2} \frac{d z^{\prime}}{H\left(z^{\prime}\right)}$,

with $z_{\text {drag }}$ being determined by the fitting formula in [55] and $\Omega_{\gamma}$ corresponding to the present photon density parameter. The data points used in the analysis are taken from [44-47].
Finally, we use the information of the CMB data from the Planck Collaboration encoded in the position of the first peak of the temperature power spectrum, $l_{1}$. The first peak at the $\mathrm{CMB}$ power spectrum can be calculated using the expression [56]:

$l_{1}=l_{A}\left\{1-0.267\left[\frac{\rho_{r}\left(z_{*}\right)}{0.3\left(\rho_{b}\left(z_{*}\right)+\rho_{d m}\left(z_{*}\right)\right)}\right]^{0.1}\right\}$,

where $l_{A}$ is the acoustic scale given by:

$l_{A}=\pi\left(1+z_{*}\right) \frac{d_{A}\left(z_{*}\right)}{r_{\mathrm{dec}}}$.

In the above expressions, $z_{*}$ is the decoupling redshift fitting in [55] and $r_{\mathrm{dec}}$ is the sound horizon scale at the decoupling epoch. We use $l_{1}=220.0 \pm 0.5$ [5].

\section{Analysis and results}

We perform a bayesian statistical analysis with the above mentioned sets of data where our posterior distribution is written in terms of the likelihood distribution, $\mathcal{L}(\Theta \mid d)$ and the prior distribution, $\pi(\Theta)$, as:

$P(\Theta \mid D) \propto \mathcal{L}(D \mid \Theta) \pi(\Theta)$,

where $\Theta$ is the set of parameters and $D$ the data considered. Our Markov Chain Monte Carlo (MCMC) simulations are made using the emcee Python module [57] assuming a Gaussian likelihood distribution,

$\mathcal{L}(D \mid \Theta) \propto \exp \left(-\chi_{T}^{2} / 2\right)$

where the total chi-square function is the sum of the contribution of each cosmological observable, $\chi_{T}^{2}=\chi_{S N e}^{2}+\chi_{B A O}^{2}+$ $\chi_{C M B}^{2}$. For the SNe Ia data we consider

$\chi_{S N e}^{2}=\left(\hat{\boldsymbol{\mu}}-\boldsymbol{\mu}_{\text {model }}\right)^{T} C_{S N}^{-1}(\alpha, \beta)\left(\hat{\boldsymbol{\mu}}-\boldsymbol{\mu}_{\text {model }}\right)^{T}$.

We also take into account statistical and systematic errors encoded in the SNe covariance matrix $C_{S N}(\alpha, \beta)$ [43].

In our statistical analysis, we use the most recent estimate of the Hubble constant $H_{0}=73.52 \pm 1.62 \mathrm{~km} \mathrm{~s}^{-1} \mathrm{Mpc}^{-1}$ 


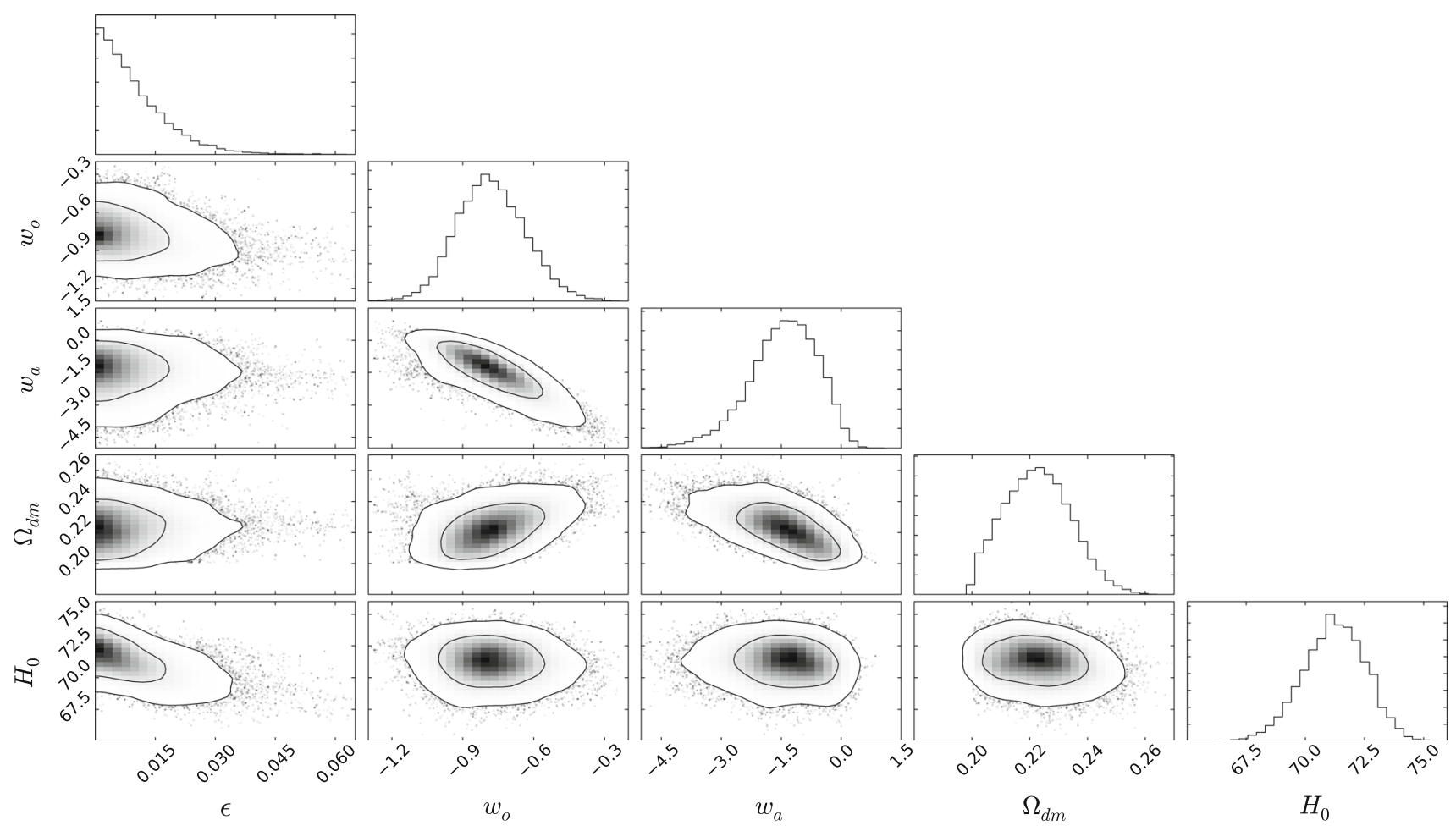

Fig. 1 The results of our statistical analysis. Confidence contours $(68.3 \%$ and $95.4 \%)$ and the posterior distribution for the cosmological parameters assuming P1 and considering the Gaussian prior $H_{0}=73.52 \pm 1.62 \mathrm{~km} \mathrm{~s}^{-1} \mathrm{Mpc}^{-1}$
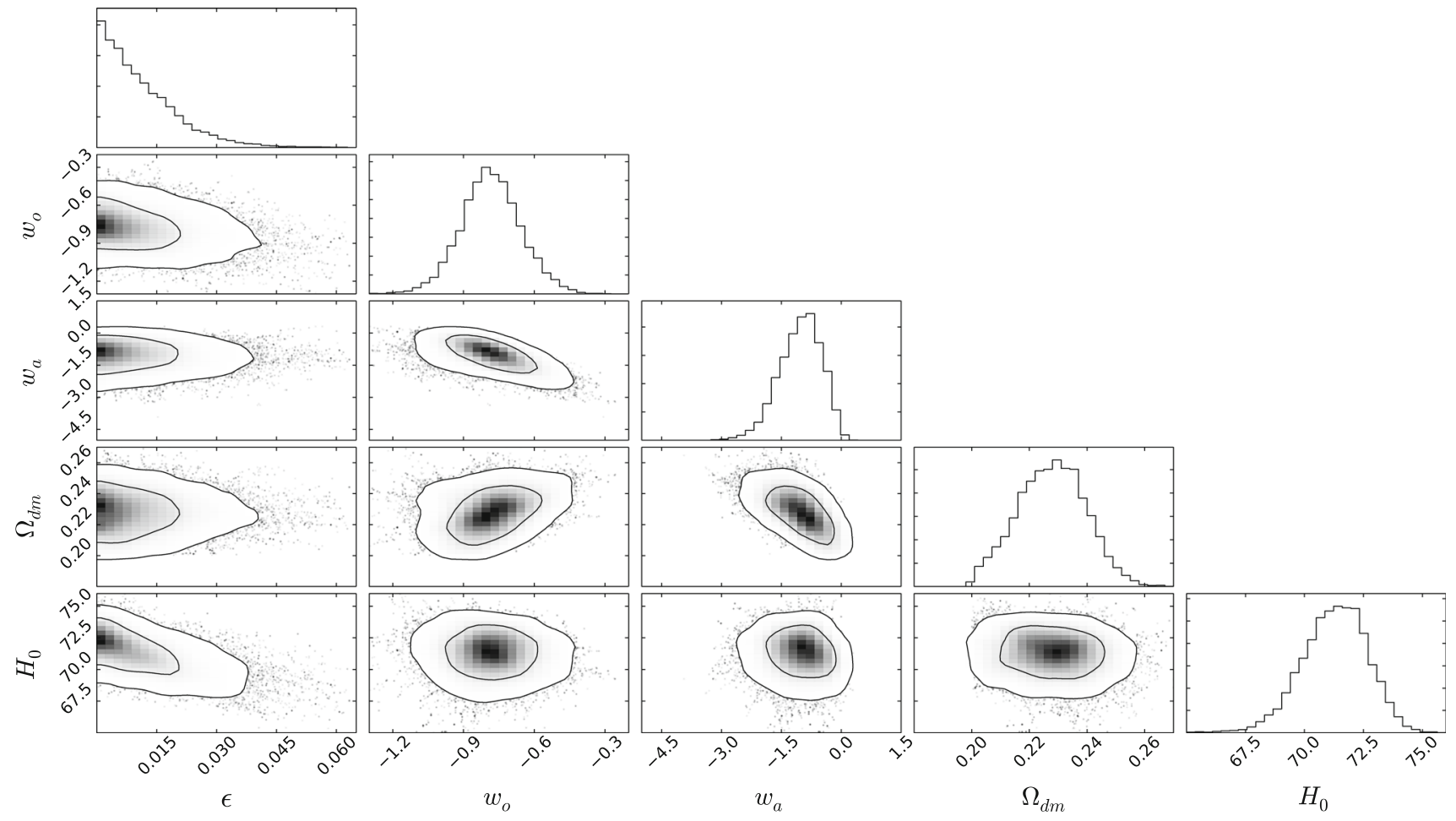

Fig. 2 The same as in Fig. 1 for P2

[49] as a Gaussian prior and flat priors for the other parameters. In particular, taking into account the constraint imposed on the $\epsilon$ parameter by [39], only positive epsilon values are allowed. We fix the baryon content at the Planck Collaboration value $\Omega_{b} h^{2}=0.02226$. The radiation density parameter used is $\Omega_{r}=4.15 \times 10^{-5} h^{-2}$ and the photon density 
Table 2 Constraints on the cosmological parameters for P1 and P2 considering a flat prior for each parameter

\begin{tabular}{llllll}
\hline & $\epsilon$ & $w_{o}$ & $w_{a}$ & $\Omega_{d m}$ & $H_{0}$ \\
\hline (P1) & $0.072 \pm 0.046$ & $-0.83 \pm 0.16$ & $-1.21 \pm 0.99$ & $0.243 \pm 0.021$ & $64.19 \pm 3.60$ \\
$(\mathrm{P} 2)$ & $0.073 \pm 0.043$ & $-0.83 \pm 0.14$ & $-0.87 \pm 0.71$ & $0.245 \pm 0.021$ & $64.19 \pm 3.31$ \\
\hline
\end{tabular}

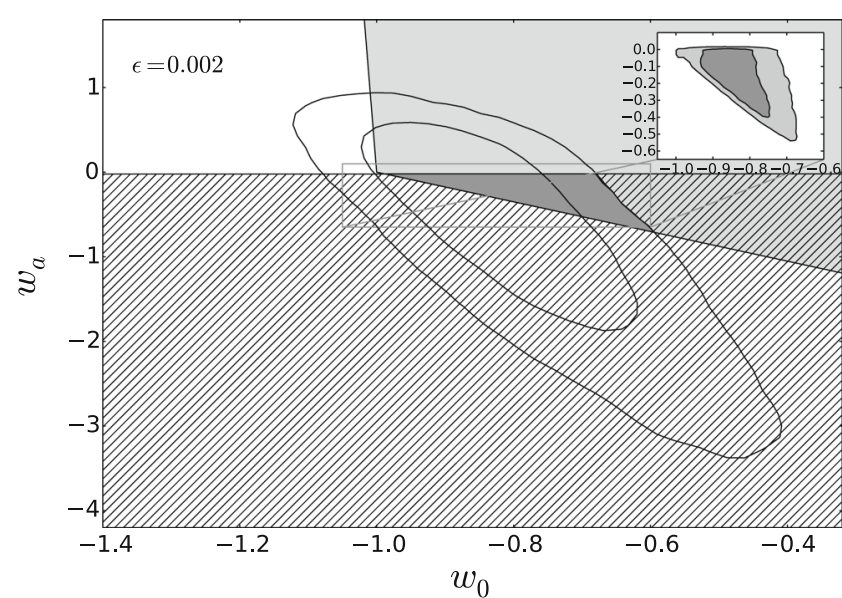

Fig. 3 Left panel Confidence contours (68.3\% and the $95.4 \%$ ) on the $w_{0}-w_{a}$ plane for parameterisation $\mathrm{P} 1$. The hatched and shaded regions correspond to the first and second sets of thermodynamic constraints, respectively. The confidence contours and the thermodynamic constraints assume $\epsilon=0.002$. Right panel The same as in the previous

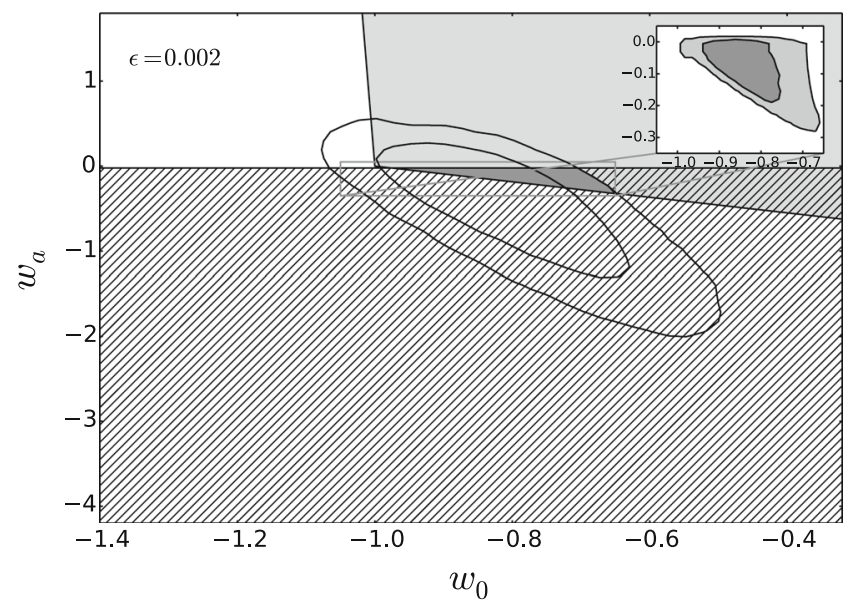

Fig. 4 The same as in Fig. 3 for $\mathrm{P} 2$ with $\epsilon=0.002$ and $\epsilon=0.02$

parameter is $\Omega_{\gamma}=2.469 \times 10^{-5} h^{-2}$ for a CMB temperature $T_{C M B}=2.725 \mathrm{~K}$.

The results of the analysis are presented in the Table 1 , and in Figs. 1 and 2 for the parameterisation P1 and P2, respectively. The figures show $1 \sigma$ and $2 \sigma$ confidence contours of the cosmological parameters and their posterior distribution marginalised over all other parameters. We also perform a statistical analysis using flat priors for the entire set of param-

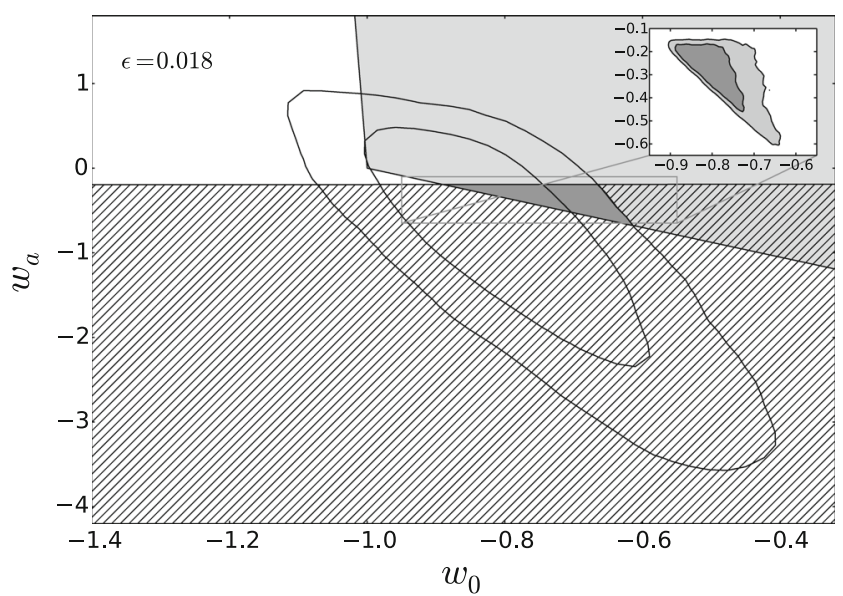

panel for $\epsilon=0.018$. The dark triangle corresponds to the combined allowed region. The subplot in each panel shows the confidence contours obtained by introducing the thermodynamical restrictions in the prior distribution $\pi(\Theta)$ to perform the statistical analysis

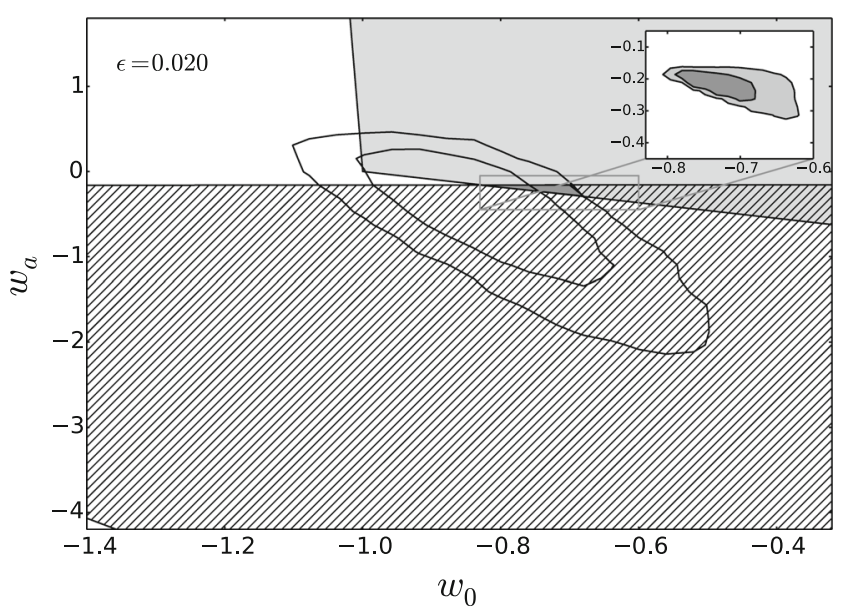

eters, whose results are presented in Table 2 . We note that the bounds on $\epsilon$ are significantly reduced when the Gaussian prior is used, in agreement with the anticorrelation between $H_{0}$ and $\epsilon$ exhibited in Figs. 1 and 2.

In order to combine the observational and the thermodynamic constraints, we also perform a statistical analysis with fixed $\epsilon$ values. We analyse the cases with $\epsilon=0.002$ and $\epsilon=0.018$ for the parameterisation P1 and with $\epsilon=0.002$ 
and $\epsilon=0.020$ for the parameterisation $\mathrm{P} 2$, which correspond to the $1 \sigma$ limits on $\epsilon$ provided by our statistical analysis (see Table 1). In Figs. 3 and 4, we present the new $1 \sigma$ and $2 \sigma$ confidence contours in the $w_{0}-w_{a}$ plane and the thermodynamic constraints (16-18) for the mentioned $\epsilon$ values. The redshift interval used in the thermodynamic constraints is $z \in(0.01,1.3)$, which corresponds to the range of the nearest and farthest $\mathrm{SNe}$ of the sample, respectively. We find that the dark energy density satisfies the weak energy condition for this $z$ interval inside the plane region in Figs. 3 and 4, therefore the second constraint set similar bounds to the ones derived in [23].

We also find that the first thermodynamic constraint is sensitive to the values of the $\epsilon$ parameter. Figures 3 and 4 show that in order to satisfy both thermodynamic conditions inside the $2 \sigma$ confidence level, the value of $\epsilon$ must be very small. Indeed, it should be smaller than the $1 \sigma$ upper limit allowed by the complete statistical analysis (see Table 1). In the analysed cases with fixed $\epsilon$ values, we find an intersection region between both thermodynamic constraints and the $2 \sigma$ observational confidence contour delimited approximately by the triangles with vertices (for P1 and P2, respectively):

$$
\begin{aligned}
& \left(w_{o}, w_{a}\right) \\
& =\left\{\begin{array}{c}
\epsilon=0.002 \\
(-0.99,-0.02),(-0.59,-0.71),(-0.68,-0.02) \\
\epsilon=0.018 \\
(-0.89,-0.19),(-0.61,-0.68),(-0.67,-0.19)
\end{array}\right. \\
& \left(w_{o}, w_{a}\right) \\
& =\left\{\begin{array}{c}
\epsilon=0.002 \\
(-0.98,-0.02),(-0.65,-0.32),(-0.71,-0.02) \\
\epsilon=0.020 \\
(-0.83,-0.16),(-0.68,-0.29),(-0.70,-0.16)
\end{array}\right.
\end{aligned}
$$

\section{Conclusions}

Relaxing the usual assumption of a minimal coupling between the components of the dark sector introduces significant changes in the predicted evolution of the universe. In this paper we have firstly discussed thermodynamic constraints on a class of interacting models assuming two parameterisations of the dark energy $\operatorname{EoS}$ (Eq. 6). The constraints on $w$ come from the second law of thermodynamics and positiveness of entropy and are combined with current observational data through a Bayesian analysis. We have shown that this combination of physical and observational constraints on $w$ impose very tight limits on the $w_{0}-w_{a}$ parametric space, as shown in Figs. 3 and 4. The thermodynamic analysis performed in this work generalises several cases previously discussed in the literature.
Acknowledgements JEG is supported by the DTI-PCI program of the Brazilian Ministry of Science, Technology, and Innovation (MCTI). RS is supported by the Conselho Nacional de Desenvolvimento Científico e tecnológico (CNPq). JSA acknowledges support from CNPq (grants no. 310790/2014-0 and 400471/2014-0) and FAPERJ (grant no. 204282).

Open Access This article is distributed under the terms of the Creative Commons Attribution 4.0 International License (http://creativecomm ons.org/licenses/by/4.0/), which permits unrestricted use, distribution, and reproduction in any medium, provided you give appropriate credit to the original author(s) and the source, provide a link to the Creative Commons license, and indicate if changes were made.

Funded by SCOAP S $^{3}$

\section{References}

1. A.G. Riess, Supernova Search Team. Astron. J. 116, 1009 (1998). arXiv:astro-ph/9805201

2. S. Perlmutter, Supernova Cosmology Project Collaboration. Astrophys. J. 517, 565 (1999). arXiv:astro-ph/9812133

3. D.J. Eisenstein, SDSS Collaboration. Astrophys. J. 633, 560 (2005). arXiv:astro-ph/0501171

4. G. Hinshaw, WMAP Collaboration. Astrophys. J. Suppl. 208, 19 (2013). arXiv:1212.5226 [astro-ph.CO]

5. P.A.R. Ade, Planck Collaboration. Astron. Astrophys. 594, A13 (2016). arXiv:1502.01589 [astro-ph.CO]

6. M. Ata, Mon. Not. Roy. Astron. Soc. 473(4), 4773 (2018). arXiv:1705.06373] [astro-ph.CO]

7. J.S. Alcaniz, J.A.S. Lima, Astrophys. J. 521, L87 (1999). arXiv:astro-ph/9902298

8. J.A.S. Lima, J.V. Cunha, J.S. Alcaniz, Phys. Rev. D 68, 023510 (2003). https://doi.org/10.1103/PhysRevD.68.023510. arXiv:astro-ph/0303388

9. M. Moresco, R. Jimenez, L. Verde, A. Cimatti, L. Pozzetti, C. Maraston, D. Thomas, JCAP 1612(12), 039 (2016). arXiv:1604.00183] [astro-ph.CO]

10. J.A.S. Lima, J.S. Alcaniz, Astron. Astrophys. 348, 1 (1999). arXiv:astro-ph/9902337

11. L.P. Chimento, A.S. Jakubi, D. Pavon, Phys. Rev. D 67, 087302 (2003). arXiv:astro-ph/0303160

12. V. Sahni, A.A. Starobinsky, Int. J. Mod. Phys. D 9, 373 (2000). arXiv:astro-ph/9904398

13. T. Padmanabhan, Phys. Rept. 380, 235 (2003). arXiv:hep-th/0212290

14. D.H. Weinberg, M.J. Mortonson, D.J. Eisenstein, C. Hirata, A.G. Riess, E. Rozo, Phys. Rept. 530, 87 (2013). arXiv:1201.2434 [astroph.CO]

15. J.A.S. Lima, J.S. Alcaniz, Phys. Lett. B 600, 191 (2004)

16. R. Silva, J.S. Alcaniz, J.A.S. Lima, Int. J. Mod. Phys. D 16, 469 (2007)

17. S.H. Pereira, J.A.S. Lima, Phys. Lett. B 669, 266 (2008)

18. R. Bousso, Phys. Rev. D 71, 064024 (2005)

19. I. Brevik, S. Nojiri, S.D. Odintsov, L. Vanzo, Phys. Rev. D 70, 043520 (2004)

20. G. Izquierdo, D. Pavon, Phys. Lett. B 633, 420 (2006)

21. D. Pavon, B. Wang, Gen. Rel. Grav. 41, 1 (2009)

22. E.M. Barboza, R.C. Nunes, E.M.C. Abreu, J.A. Neto, Phys. Rev. D 92(8), 083526 (2015)

23. R. Silva, R.S. Goncalves, J.S. Alcaniz, H.H.B. Silva, A\& A 537, A11 (2012)

24. H.H.B. Silva, R. Silva, R.S. Gonalves, Z.H. Zhu, J.S. Alcaniz, Phys. Rev. D 88, 127302 (2013)

25. S. Weinberg. arXiv:astro-ph/0005265 
26. H.E.S. Velten, R.F. vom Marttens, W. Zimdahl, Eur. Phys. J. C 74(11), 3160 (2014). arXiv:1410.2509 [astro-ph.CO]

27. L. Amendola, Phys. Rev. D 60, 043501 (1999)

28. L. Amendola, Phys. Rev. D. 62, 043511 (2000)

29. W. Zimdahl, D. Pavon, Phys. Lett. B 521, 133 (2001)

30. C.G. Boehmer, G. Caldera-Cabral, R. Lazkoz, R. Maartens, Phys. Rev. D 78, 023505 (2008)

31. L.P. Chimento, M.G. Richarte, Phys. Rev. D 85, 127301 (2012)

32. F.E.M. Costa, J.S. Alcaniz, D. Jain, Phys. Rev. D 85, 107302 (2012). arXiv:1204.3066 [astro-ph.CO]

33. R. F. vom Marttens, L. Casarini, W.S. Hipólito-Ricaldi, W. Zimdahl, JCAP 1701(01), 050 (2017). arXiv:1610.01665 [astroph.CO]

34. R.F. vom Marttens, L. Casarini, W. Zimdahl, W.S. HipólitoRicaldi, D.F. Mota, Phys. Dark Univ. 15, 114-124 (2017). arXiv: 1702.00651 [astro-ph.CO]

35. R. von Marttens, L. Casarini, D.F. Mota, W. Zimdahl. arXiv: 1807.11380 [astro-ph.CO]

36. F. Arevalo, A. Cid, J. Moya, Eur. Phys. J. C 77(8), 565 (2017)

37. A. Cid, B. Santos, C. Pigozzo, T. Ferreira, J. Alcaniz. arXiv:1805.02107 [astro-ph.CO]

38. P. Wang, X.H. Meng, Class. Quant. Grav. 22, 283 (2005)

39. J.S. Alcaniz, J.A.S. Lima, Phys. Rev. D 72, 063516 (2005)

40. M. Chevallier, D. Polarski, Int. J. Mod. Phys. D 10, 213 (2001)

41. E.V. Linder, Phys. Rev. Lett. 90, 091301(2003)
42. E.M. Barboza Jr., J.S. Alcaniz, Phys. Lett. B 666, 415 (2008)

43. M.R. Betoule et al., A\&A 568, A22 (2014)

44. G.C. Carvalho, A. Bernui, M. Benetti, J.C. Carvalho, J.S. Alcaniz, Phys. Rev. D 93(2), 023530 (2016)

45. J.S. Alcaniz, G.C. Carvalho, A. Bernui, J.C. Carvalho, M. Benetti, Fundam. Theor. Phys. 187, 11 (2017)

46. G. C. Carvalho, A. Bernui, M. Benetti, J. C. Carvalho, J. S. Alcaniz. arXiv:1709.00271 [astro-ph.CO]

47. E. de Carvalho, A. Bernui, G.C. Carvalho, C.P. Novaes, H.S. Xavier, JCAP 1804(04), 064 (2018)

48. P.A.R. Ade et al., A\&A 594, A13 (2016)

49. A.G. Riess et al., Astrophys. J. 861(2), 126 (2018)

50. C. Escamilla-Rivera, L. Casarini, J.C. Fabris, J.S. Alcaniz, JCAP 1611(11), 010 (2016). arXiv:1605.01475] [astro-ph.CO]

51. J.S. Alcaniz, H.A. Borges, S. Carneiro, J.C. Fabris, C. Pigozzo, W. Zimdahl, Phys. Lett. B 716, 165 (2012). arXiv:1201.5919 [astroph.CO]

52. S. Weinberg, Astrop. J. 168, 175 (1971)

53. R. Silva, J.A.S. Lima, M.O. Calvão, Gen. Rel. Grav. 34, 865 (2002)

54. G.R. Farrar, P.J.E. Peebles, Astrophys. J. 604, 1 (2004)

55. W. Hu, N. Sugiyama, Astrophys. J. 471, 542 (1996)

56. W. Hu et al., Astrophys. J. 549, 669 (2001)

57. D. Foreman-Mackey, D.W. Hogg, D. Lang, J. Goodman, Publ. Astron. Soc. Pacif. 125, 925 (2013) 